\title{
Guía de Esperanza del Programa Abriendo Oportunidades
}

Population Council

Follow this and additional works at: https://knowledgecommons.popcouncil.org/departments_sbsr-pgy How does access to this work benefit you? Let us know!

\section{Recommended Citation}

"Guía de Esperanza del Programa Abriendo Oportunidades." Ciudad de Guatemala: Population Council, 2021. 


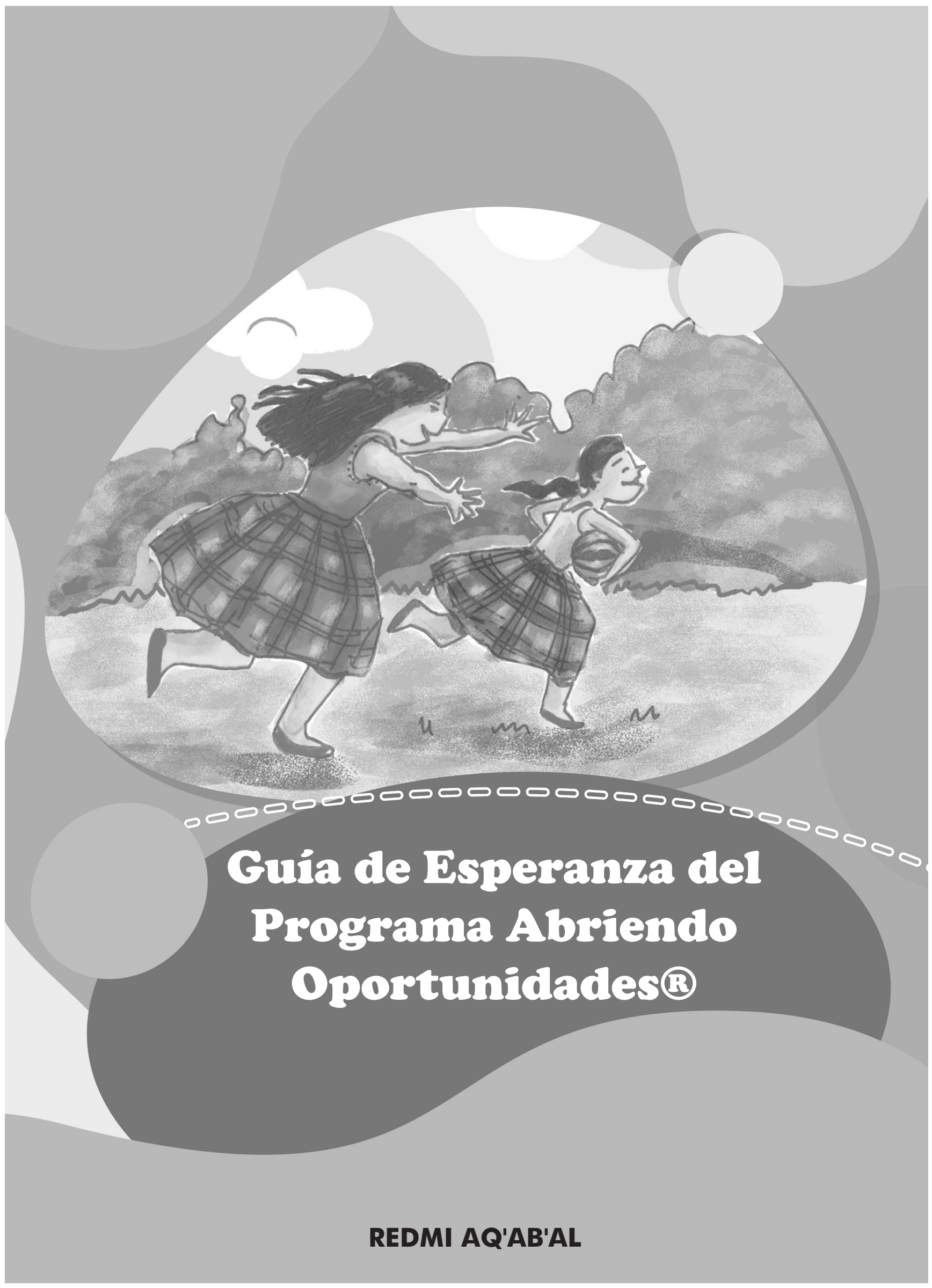




\section{Guía de Esperanza del Programa Abriendo Oportunidades ${ }^{\circledR}$}
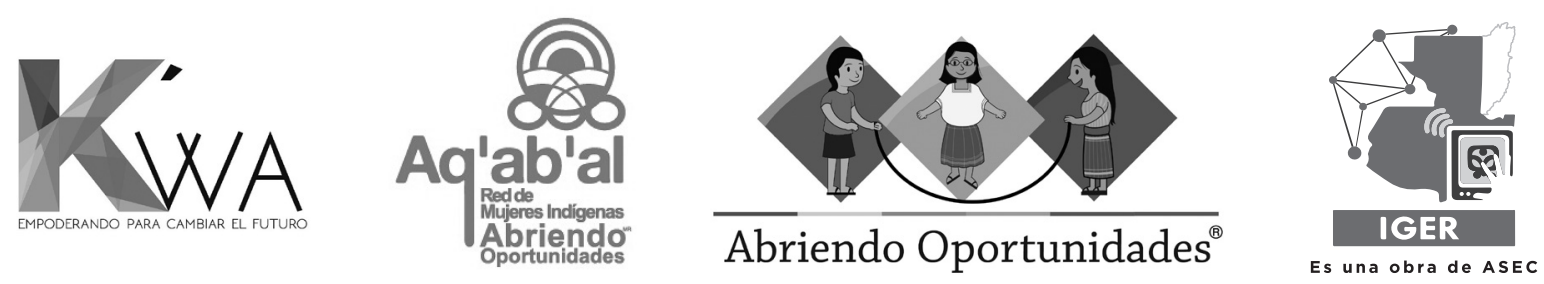

La asociación Red de Mujeres Indígenas Abriendo Oportunidades ${ }^{\circledR}$ es una entidad integrada por mujeres jóvenes mayas. REDMI está integrada por mentoras graduadas de Abriendo Oportunidades. Su objetivo de REDMI es proveer a niñas y adolescentes en comunidades rurales un espacio seguro para aprender sobre su vida, fortalecer sus redes de apoyo y amistad con otras niñas, y equiparlas con habilidades para la vida.

Contacto: red.mujeres.gt@gmail.com

Facebook: REDMI Aq'ab'al

Contenido: Abriendo Oportunidades ${ }^{\circledR}$

Mediación: Sandra López

Diagramación: Linda Tenas

llustración: Armando Cruz y Linda Tenas

Edición 2020

Impreso en IGER talleres gráficos

Código: 6131251102

ISBN: 978-9929-766-63-1

Guatemala, 2020

Con el apoyo financiero de Asociación K'wa. 


\section{Índice}

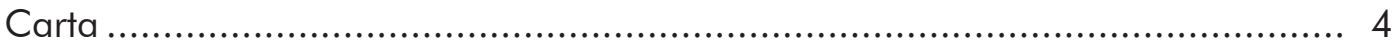

\section{Tema I}

Abriendo oportunidades............................................................... 5

\section{Terma 2}

Derechos humanos

\section{Tema 3}

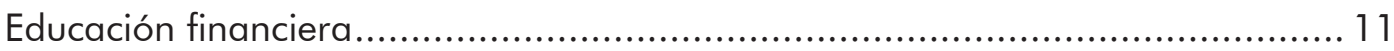

\section{Tema 4}

Género

\section{Tema 5}

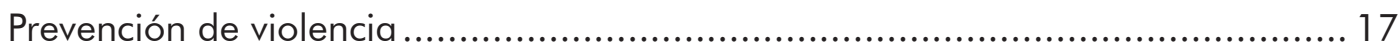

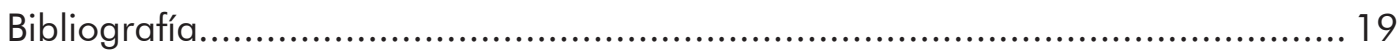




\section{Queridas iMentoras!}

Les escribimos por este medio para desearles éxitos en la nueva fase de implementación del programa radial Abriendo Oportunidades ${ }^{\circledR}$ en tu comunidad. Esperando que se encuentren bien y sus familias.

Estamos experimentado situaciones inesperadas por el confinamiento del COVID-19 sin embargo, tenemos que seguir trabajando en equipo con una actitud positiva en pro del empoderamiento de las niñas y adolescentes que viven en las comunidades rurales. Hoy más que nunca necesitan de ustedes como mentoras comunitarias para brindar información y orientación adecuada, principalmente en temas de salud, educación en derechos humanos, prevención

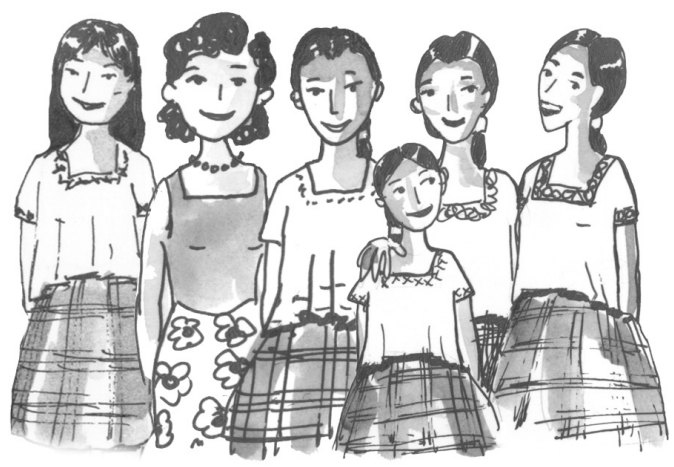
de violencia, equidad de género entre otros.

La presente guía fue elaborada como herramienta para desarrollar temas dirigido a niñas y adolescentes ante la pandemia, es importante equiparnos en habilidades y destrezas, posteriormente proveerles herramientas estratégicas que les permite generar nuevos conocimientos y experiencias.

Estamos seguras, que, con su dedicación y esfuerzo, lograremos que las niñas $y$ adolescentes reciban el contenido del programa radial.

iiAbrazos!!

REDMI AQ'AB'AL 


\section{Abriendo oportunnidades

Dé la bienvenida a las participantes.

\section{Materiales a utilizar}

- Cuaderno de apuntes de las participantes

- Lapiceros y crayones

- Libro: Abriendo oportunidades

- Redes sociales y/o correo electrónico.

- Teléfono

- Radio

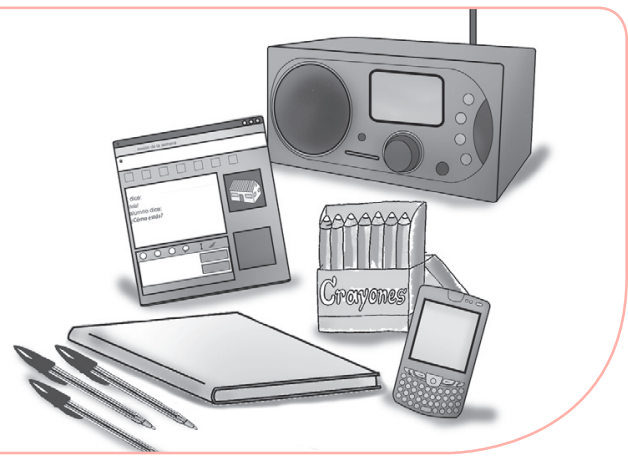

\section{Palabras clave}

En este espacio encuentra los principales conceptos que se deben fortalecer con las participantes. Es importante que los lea y elabore ejemplos de cada uno, de acuerdo a las edades de las participantes.

\section{Espacio seguro:}

Es el lugar y tiempo en el que las niñas adolescentes se sienten seguras, protegidas para aprender, compartir y formar una red de amigas en las que se apoyan.

\section{Convivencia:}

Relación respetuosa de la dignidad de las personas, en la que puede iniciar una nueva amistad. Para ello puede utilizar las redes sociales y correo electrónico.

Resilencia: capacidad que tiene una persona o una comunidad para superar circunstancias traumáticas.

Autoestima: sentimiento de aceptación y aprecio hacia uno mismo. Se fortalece en la medida que se alcanzan pequeños y grandes logros. 


\section{Conocimientos previos}

Pida a las participantes que escriban en su cuaderno cuáles son sus expectativas o qué esperan aprender en este programa.

Motive a las participantes a que las publiquen en sus redes sociales y/o correo electrónico.

Diga a las participantes:

Todas tenemos algo especial y todas somos bienvenidas al escuchar el programa radial de Abriendo Oportunidades ${ }^{\circledR}$. Es importante que nuestra casa sea un espacio donde podamos soñar, cometer errores, aprender y lograr nuestras metas.

Cuando escuche el programa radial, tenga listos todos sus materiales: su libro y cuaderno de apuntes.

\section{Actividades}

- Motive a las participantes a leer, en silencio, su biografía.

- Explique en qué consiste la resilencia.

- Explique el concepto de autoestima.

- Solicite a las participantes a que realicen el siguiente cuadro, escribiendo al menos tres características en cada aspecto.

\begin{tabular}{|l|l|}
\hline Cualidades mías que aprecio yo & Cualidades mías que aprecian los demás \\
\hline & \\
\hline & \\
\hline & \\
\hline
\end{tabular}

- Pida que dibujen sus sueños y metas.

\section{Compartir lo aprendido}

Motive a las participantes a que escriban en este cuadro, así como el dibujo de sus sueños y metas.

Solicite a las participantes que investiguen la biografía de una mujer, preferiblemente que sea de su comunidad, que ha aplicado la resilencia en alguna etapa de su vida. 


\section{Conocimientos previos}

Pídales a las participantes que respondan las preguntas que se presentan en el libro Abriendo Oportunidades, tema 1 y 2 . Presente un ejemplo de desigualdad cuando lea cada una de ellas.

¿Los niños y niñas tienen los mismos derechos humanos?

¿Los niños y niñas son tratados de la misma forma en su comunidad?

¿Todos los niños y niñas van a la escuela?

Las mujeres y los hombres que realizan el mismo trabajo ¿̇tienen el mismo pago?

¿Se trata igual a los niños y niñas en el hogar?

Reflexione con las participantes que cada derecho trae una responsabilidad. Y que los derechos humanos se aplican a todas las personas sin distinción de raza, sexo, nacionalidad, origen étnico, lengua, religión o cualquier otra condición.

\section{Actividades}

- Enfatice en la importancia de conocer sus derechos.

- Solicite a las participantes que realicen un listado de todas las medidas de prevención para evitar contagiarse del COVID-19.

Pueden apoyarse en la información que dan en la televisión, en la radio o recibido en otros medios. Por ejemplo, lavarse las manos constantemente.

- Pida a las participantes que escriban cómo se aplican los derechos humanos en su hogar, comunidad y país, en estos momentos que se está viviendo la pandemia por COVID-19. Por ejemplo, todos los miembros de su familia se protegen y cuidan unos a otros lavándose las manos y utilizando mascarilla.

\section{Derechos:}

Grupo 1. Todas las niñas y niños tienen el derecho a tener un nombre y apellido.

Grupo 2. Todas las niñas y niños tienen el derecho a tener una familia que le cuida y quiera.

Grupo 3. Todas las niñas y niños tienen el derecho a tener una vivienda digna en donde vivir.

Grupo 4. Todas las niñas y niños tienen el derecho a ir a la escuela para aprender cosas interesantes e importantes para su vida.

Grupo 5. Todas las niñas y niños tienen el derecho a recibir atención médica para cuidar su salud.

\section{Compartir lo aprendido}

Pida a las participantes que anoten en un cuaderno lo aprendido de la sesión radial.

Motive a las participantes a que elaboren sus propias conclusiones de la actividad y que las comparta con su familia. 


\section{COVID-19}

CORONAVIRUS

\section{Medidas de PReVención}

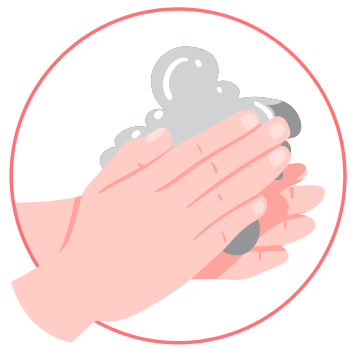

Lávese las manos constantemente

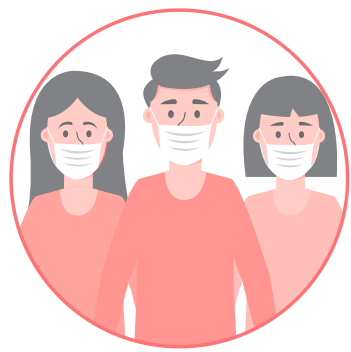

Evite lugares concurridos

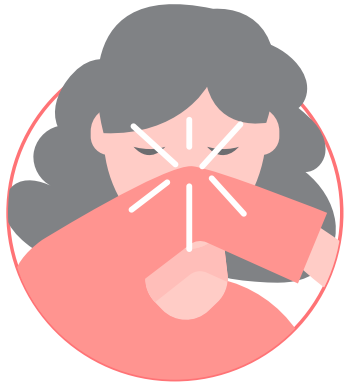

Cubrase al toser o estornudar

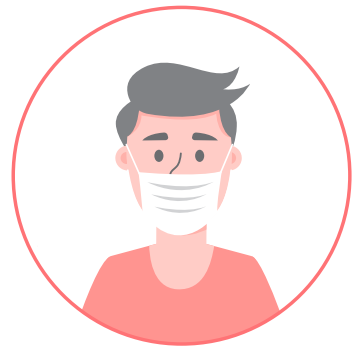

Use mascarilla

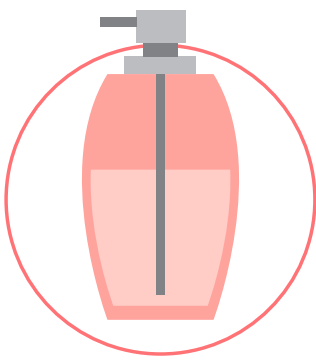

Utilice gel antibacterial

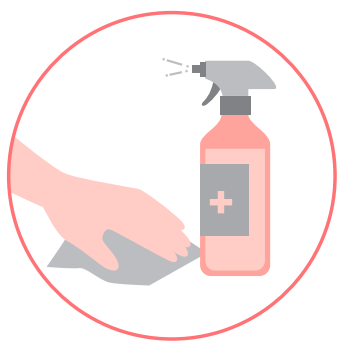

Desinfecte superficies 



\section{Educación financiera}

Dé la bienvenida a las participantes.

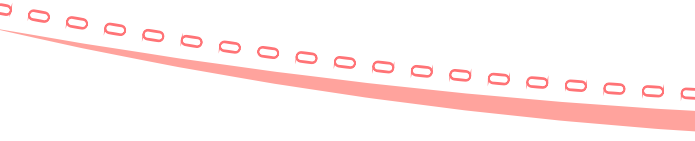

En este espacio encuentra los principales conceptos que se deben fortalecer durante el proceso de aprendizaje. Es importante que los lea y elabore ejemplos de cada uno, de acuerdo a las edades de las participantes.

\section{Necesidad:}

Lo que se debe tener para vivir de forma digna y saludable, puede ser material o inmaterial.

\section{Deseo:}

Son bienes o servicios que se ansían sin que satisfagan alguna necesidad básica.

\section{Utilidad:}

Acciones pensadas que evitan el riesgo de daño al bienestar de la persona.

\section{Selección:}

Capacidad de escoger entre una cosa u otra cosa según su utilidad de acuerdo con las necesidades y deseos.

\section{Ahorro:}

Dinero y/o recurso que se preserva sin ser consumido para acumularlo con otros similares que sean de gran utilidad para tener mayor capacidad de consumo.

\section{Presupuesto:}

Asignar la cantidad de dinero en diversos rubros. Distribución de los ingresos en gastos. 


\section{Conocimientos previos}

Motive a las participantes que vuelvan a leer, en su cuaderno de trabajo, todas las cosas que quieren hacer en su vida.

Realice la siguiente pregunta: ¿Cómo pueden llegar a obtener los recursos que necesitan para lograrlas?

Invite a las participantes a reflexionar acerca de cómo pueden obtener los recursos que necesitan.

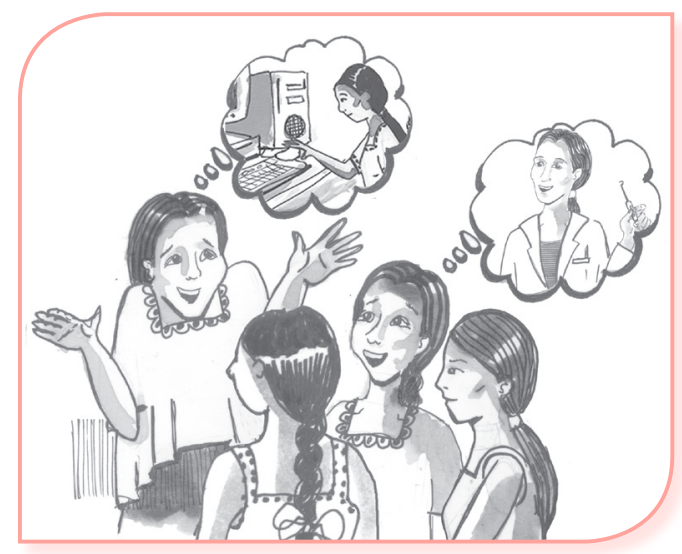

\section{Actividades}

- Explique acerca de la importancia de administrar el dinero por medio de un presupuesto.

- Dibuje en el portafolio este formato, el cual utilizarán las participantes para elaborar el listado de gastos mensuales familiares y personales.

\begin{tabular}{|l|l|}
\hline \multicolumn{1}{|c|}{ Tipo de gasto } & \multicolumn{1}{|c|}{$\begin{array}{c}\text { Cantidad de dinero que se } \\
\text { gasta mensualmente }\end{array}$} \\
\hline Comida & Q. 800.00 \\
\hline & \\
\hline
\end{tabular}

Pida a las participantes que elaboren un listado (sin escribir la cantidad de dinero) de todos los gastos que se realizan en su hogar mensualmente. (Por la edad de las participantes, estos gastos los realizan los padres de familia) Por ejemplo, vivienda, servicio de agua, etc.

Solicite a las participantes que realicen otro listado con todos sus gastos personales (sin escribir la cantidad de dinero). Por ejemplo, transporte, comida, luz, agua, pasta de dientes, etc.

Explique que hay algunos gastos que se realizan todos los días y otros una vez al mes.

Por ejemplo, si gasta Q. 4.00 para ir a la escuela o al trabajo cada día, debe multiplicarlo por los 20 días que asiste al mes:

Q. $4.00 \times 20=$ Q. 80.00 mensuales.

\begin{tabular}{|l|c|}
\hline \multicolumn{1}{|c|}{ Tipo de gasto } & $\begin{array}{c}\text { Cantidad de dinero que se } \\
\text { gasta mensualmente }\end{array}$ \\
\hline Transporte & Q. 80.00 \\
\hline Vivienda (propia o alquiler) & Q. 2000.00 \\
\hline
\end{tabular}


- Oriente a las participantes a elaborar los cálculos de su presupuesto.

- Explique la importancia del ahorro, de distribuir adecuadamente la cantidad de dinero que recibimos por medio de un salario $u$ otro ingreso y de evitar adquirir deudas innecesarias.

- Solicite la participación voluntaria de una participante para leer su presupuesto personal.

\section{Compartir lo aprendido}

Motive a las participantes a que elaboren sus propias conclusiones de la actividad y las compartan a con la persona más cercana de su confianza. 



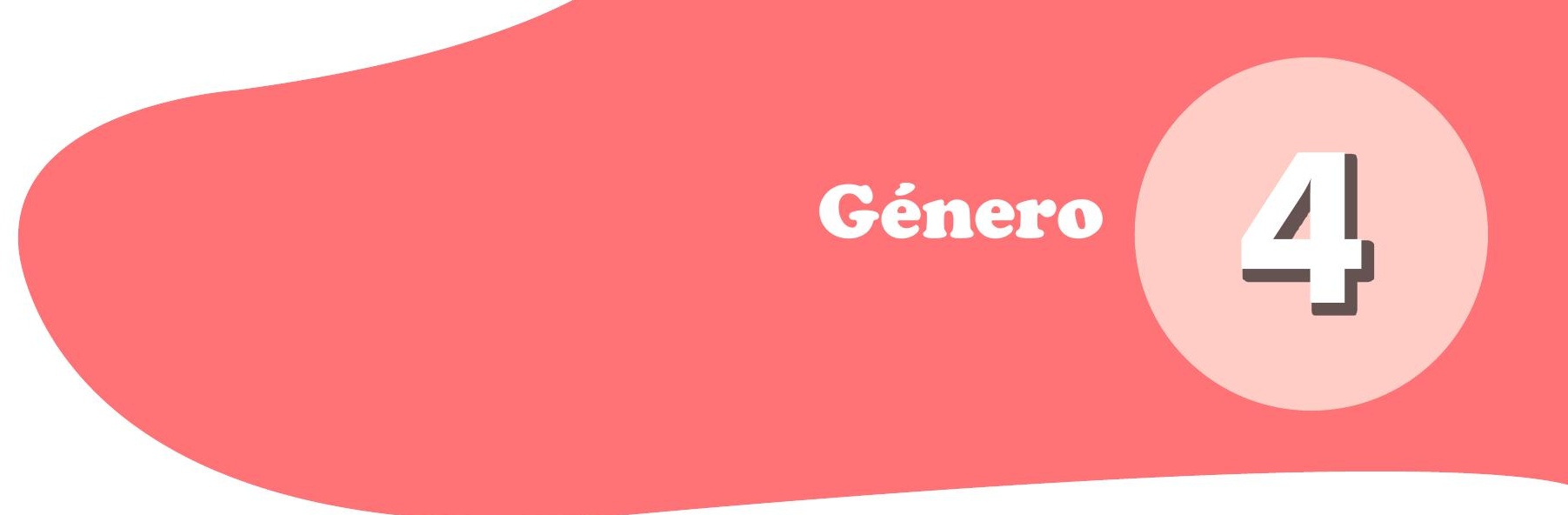

Dé la bienvenida a las participantes.

\section{Palabras clave}

En este espacio encuentra los principales conceptos que se deben fortalecer. Es importante que los lea y elabore ejemplos de cada uno, de acuerdo a las edades de las participantes.

\section{Sexualidad:}

La sexualidad comprende nuestro cuerpo, nuestra forma de sentir, de pensar y de ser en una sociedad y en una época determinada. La sexualidad abarca las ideas, los pensamientos, las palabras, los deseos, las emociones, los valores y los comportamientos, es decir, todo nuestro ser.

\section{Género:}

Son las características y conductas que se asignan a hombres y mujeres por parte de su comunidad y familia. La sociedad asigna roles, valores y comportamientos diferentes a cada uno.

\section{Equidad de género:}

Los hombres y mujeres tienen los mismos derechos de realizar cualquier actividad como estudiar y ganar el mismo salario al realizar un mismo trabajo.

\section{Conocimientos previos}

Motive a las participantes para que respondan las siguientes preguntas:

¿̇Los niños y las niñas en su hogar hacen las mismas tareas? (oficio, cocinar, lavar trastos, otros)

¿Qué diferencias existen entre las actividades que hacen los niños y niñas en la escuela, en los deportes o juegos que realizan?

Piense en cómo era su mamá y su abuelita cuando tenían la misma edad que usted. ¿̇Ellas escuchaban la misma música, usaban ropa y zapatos parecidos a los que usted usa? 
La forma en que educaron a su papá y a su mamá żes la misma forma en que sus padres la están educando a usted y a sus hermanas y hermanos?

¿Existen algunos aspectos en su vida que desea cambiar?

\section{Actividades}

Apoye a las participantes a analizar el siguiente cuadro.

Realice las siguientes preguntas: ¿̇Cómo se llegan a dar tantas diferencias entre niños y niñas?

¿De dónde aprendemos estas diferencias?

Enfatice el concepto de equidad de género.

\begin{tabular}{|l|l|}
\hline \multicolumn{1}{|c|}{ Las niñas... } & \multicolumn{1}{|c|}{ Los niños... } \\
\hline $\begin{array}{l}\text { No necesitan estudiar porque nacieron } \\
\text { para casarse. }\end{array}$ & $\begin{array}{l}\text { Tienen que prepararse para el futuro, por } \\
\text { eso deben ir a la escuela. }\end{array}$ \\
\hline Se visten de rosado. & Se visten de celeste. \\
\hline $\begin{array}{l}\text { No tienen tiempo de jugar. Tienen que } \\
\text { ayudar a su mamá en la casa. }\end{array}$ & Tienen derecho a jugar y tener amigos. \\
\hline Pueden llorar y expresar sus emociones. & No deben llorar o expresar emociones. \\
\hline Juegan con muñecas, cocina y trastos. & $\begin{array}{l}\text { Juegan con carros de bomberos, pelotas, } \\
\text { juegos de doctor. }\end{array}$ \\
\hline
\end{tabular}

- Las siguientes frases:

$\checkmark$ Las niñas son más indefensas y por eso deben estar siempre acompañadas de un hombre que las pueda cuidar.

$\checkmark$ Las mujeres que salen de la comunidad y de casa a trabajar ayudan al desarrollo de su familia.

$\checkmark$ Cuando las niñas se vuelven señoritas no deben estar saliendo solas por allí en la calle.

$\checkmark$ Las niñas tienen derecho a estudiar e ir a la universidad.

$\checkmark$ La tierra la heredan los hijos para mantenerla en la familia

- Motive a las niñas que se reúnan en grupos de tres y que analicen entre ellas, si cada una de las frases ayudan o limitan el desarrollo de las niñas.

- Solicite a las participantes para que dibuje una carita alegre a la par de las frases que ayudan al desarrollo de las niñas.

- Recuerde los temas vistos en el cuaderno de trabajo y solucione las dudas que puedan tener las participantes.

\section{Compartir lo aprendido}

Motive a las participantes a que elaboren sus propias conclusiones de la actividad y las compartan a las niñas a las mujeres de su alrededor. 


\section{Prevención de la violencia}

\section{Dé la bienvenida a las participantes.}

\section{Palabras clave}

En este espacio encuentra los principales conceptos que se deben fortalecer durante el proceso de aprendizaje. Es importante que los lea y elabore ejemplos de cada uno, de acuerdo a las edades de las participantes.

Lugar de riesgo:

Espacio en el que puede suceder algo que produzca un daño físico, emocional, psicológico o de cualquier otro tipo. No hay personas que ofrezcan protección ni apoyo.

Comportamiento riesgoso:

Aquellas acciones que ponen en peligro el bienestar de la persona, propiciando el sufrimiento de algún tipo de daño.

Prevenir:

Acciones pensadas que evitan el riesgo de daño al bienestar de la persona.

Abuso:

Cualquier comportamiento que utiliza una persona para controlar a otra, hace uso del poder, miedo, intimidación o seducción. Puede haber abuso emocional o psicológico y abuso sexual. 


\section{Conocimientos previos}

Diga a las participantes que elaboren un listado de los lugares de su comunidad donde se sienten seguras. Puede incluir la casa de algunos amigos y familiares.

Pida a las participantes que identifiquen los lugares de su comunidad donde ellas piensan que corren algún tipo de peligro y que escriban por qué se sienten inseguras.

Comparta con las participantes que, para estar seguras, es importante conocer los lugares de la comunidad para saber a dónde ir según las necesidades que se tengan o para hacer los mandados que son necesarios.

\section{Actividades}

- Motive a las participantes a que reflexionen de las siguientes situaciones, aquellas donde corren algún tipo de riesgo.

$\checkmark$ Ir acompañada de una amiga cuando voy por caminos solitarios.

$\checkmark$ Ir a la tienda solita por la tarde-noche a comprar un dulce.

$\checkmark$ Hablar con personas que no conozco pero parecen amables.

$\checkmark$ Dejar dicho en mi casa a dónde voy, con quién voy y cuánto tiempo voy.

$\checkmark$ Decir que voy al mercado pero después cambiar de lugar sin avisar a nadie.

- Explique a las participantes que identifiquen situaciones de riesgos y que conozcan los lugares de la comunidad que representan peligros, le ayudan a tomar las medidas de precaución necesarias.

Conocer los lugares significa no sólo saber en dónde quedan, sino que también conocer qué pasa en esos lugares, qué personas van allí, cómo las reciben y tratan y qué tipo de riesgo se corre.

- Invite a las participantes a que elaboren un listado de las instituciones que existen en su comunidad y que pueden ayudarle si es víctima de algún tipo de violencia. Por ejemplo, la Policía Nacional Civil.

Si no conocen las instituciones que pueden proporcionarle ayuda en su comunidad, motívelas a que investiguen cuáles son.

\section{Compartir lo aprendido}

Ayude a las participantes a elaborar sus conclusiones.

Motive a las participantes a que compartan con otras mujeres las medidas de prevención de la violencia contra la mujer y los lugares de su comunidad donde pueden pedir ayuda y denunciar. 


\section{BIBLIOGRAFIA}

IGER. (2017). Biología, grupo Polochic. Guatemala: IGER.

MINEDUC, UNFPA, UNESCO. (2016) Fascículo 9, Educación integral en sexualidad y prevención de la violencia. Prevención de la violencia.

IGER. (2016). Ciencias Naturales, grupo Quiriguá. Guatemala: IGER.

IGER, UNFPA, UNESCO. (2016) Un viaje desde mi interior hacia mi futuro. Educación integral en sexualidad. Guatemala: IGER.

POPULATION COUNCIL, UNFPA. (2016) Abriendo Oportunidades: Guía Curricular Integrada. Módulo 1. Guatemala: Population Council. 
Apuntes importantes: 